\title{
Survey on Discovering rapid Changing Patterns In the presence of Hierarchy
}

\author{
Priya Sahane ${ }^{1}$, Prof.A.N. Nawathe ${ }^{2}$ \\ ${ }^{\prime}$ (Computer, AVCOE/ Pune, India) \\ ${ }^{2}$ (Computer, AVCOE/ Pune,Indiae)
}

\begin{abstract}
Frequent item set mining is used that focuses on find out recurrent correlations in the data. Change mining, it focuses on frequent itemsets, focuses on important changes in the set of mined itemsets from one point in time period to another. The finding of frequent generalized itemsets, One dynamic pattern, the history generalized pattern, that represents the development of an itemset in successive time periods, by accounting the information about its recurrent generalizations characterized by minimal redundancy some time it becomes infrequent. Higen mining, The higen miner, that focuses on avoiding itemset mining followed by postprocessing by developing a support-driven itemset generalization .To focus the attention on the minimally redundant recurrent generalizations and reduce the amount of the generated patterns, the finding a subset of higens, namely the nonredundant higens, . Tests do on both real and synthetic datasets show the competence and the effectiveness [1] .
\end{abstract}

Keywords: Data mining algorithm, Data mining Method;

\section{INTRODUCTION}

Frequent itemset mining algorithm is constrained by a minimum support threshold to discover patterns whose observed support in the source data is equal to or surpasss a given threshold [2]. The put into forcefully of low support thresholds may involve generating a very huge amount of patterns which may turn out to be difficult see in to that this is case when support count value is less than given threshold.In another case higher threshold forcefully inserted into the dataset might be prune relevant but not sufficient frequent recurrences.

Generalized itemsets, which have been first introduced in [4] in the context of market basket analysis, are itemsets that provide a high level abstraction of the mined knowledge. Tables 1 and 2 represent two example of datasets, related with two following months of year 2013 June and July. Each record be in contacts to a product sale \& the good description, the date, the time, and the location in which position are reported. Fig. 1 shows a simple tree structure defined on the location attribute. Table 1 reports the set of all probable frequent generalized and not generalized itemsets, and their equivalent absolute support values mined, [4], from the example datasets D1 and D2,by exploiting the tree reported in Fig. 1 and by implementing an absolute minimum support threshold equal to 2. In both dataset that is D1 and D2 itemset are suit the support threshold and other are frequent in one months out of two months. In the case of frequent itemsets by exploiting generalized itemsets to represent patterns that become unusual with respect to the support threshold, and therefore they are no longer extracted, at a specific point. [3], [8], [9].This type of algorithm is used to discovering basic changes [6], [13], [14]).

Table 1

(a)Dataset D1. Good sales in June 2013

\begin{tabular}{|l|l|l|l|}
\hline Date & Time & Location & Product \\
\hline $\begin{array}{l}2013-06- \\
02\end{array}$ & $\begin{array}{l}11: 15 \\
\text { p.m. }\end{array}$ & Bihar & Paint \\
\hline $\begin{array}{l}2013-06- \\
02\end{array}$ & $\begin{array}{l}10: 28 \\
\text { p.m. }\end{array}$ & Bihar & Paint \\
\hline $\begin{array}{l}2013-06- \\
05\end{array}$ & $\begin{array}{l}8: 51 \\
\text { a.m. }\end{array}$ & Paris & Jacket \\
\hline $\begin{array}{l}2013-06- \\
05\end{array}$ & $\begin{array}{l}5.05 \\
\text { p.m. }\end{array}$ & Cannes & Jacket \\
\hline $\begin{array}{l}2013-06- \\
28\end{array}$ & $\begin{array}{l}5.00 \\
\text { p.m. }\end{array}$ & Asamm & Jacket \\
\hline
\end{tabular}

(b)Dataset D2. Good sales in Jule 2013

\begin{tabular}{|c|c|c|c|}
\hline Date & Time & Location & Good Description \\
\hline $\begin{array}{l}2013-07- \\
02\end{array}$ & $\begin{array}{l}11: 00 \\
\text { p.m. }\end{array}$ & Bihar & Paint \\
\hline $\begin{array}{l}2013-07 . \\
03\end{array}$ & $\begin{array}{l}\text { 11:10 } \\
\text { pm. }\end{array}$ & Asam & Paint \\
\hline $\begin{array}{l}2013-07- \\
05\end{array}$ & $8: 40 \mathrm{am}$. & Panis & Jacket \\
\hline $13-07-30$ & $5.05 \mathrm{pm}$. & Panis & Jacket \\
\hline $\begin{array}{l}2013-07- \\
31\end{array}$ & $5.00 \mathrm{pm}$ & Cannes & Jacket \\
\hline
\end{tabular}

Consider the itemsets \{Paint, Bihar\} and \{Jacket, Paris\}. The former itemset is nonfrequent in D1 means its support count value in D1 is lower than the support threshold and frequent itemset in D2 support value increase from June to July, after that shows an opposite.The discovery of higher level relationship, in the form of generalized itemsets,. Item set generalization allowed only avoiding infrequent itemset. At the same time, the information is provided by the series of generalizations or specializations of the same pattern in successive time durations. The generalized itemset \{Paint, India\}has a specialization \{Paint, Bihar\}) that is 
infrequent in June and frequent in the july might be relevant for decision making and,reported as a temporal correlation among Recurrences about product Paint. Same as, the information that, although \{Jacket, Paris\} infrequent with respect to the minimum support threshold transferring from June to July, its generalization \{Jacket, France\}remains frequent in both months can be relevant for analyst decision making. this type of information cannot be directly mined or to the point represented means of any existing concept.

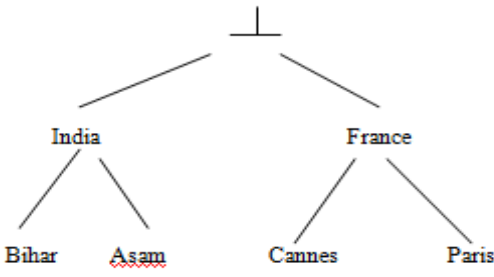

Fig. 1. Generalization tree structure. for the location attribute in the datasets $\mathrm{D} 1$ and $\mathrm{D} 2$.

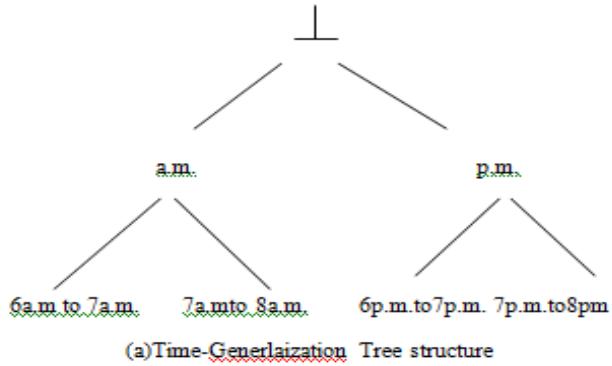

Table 2

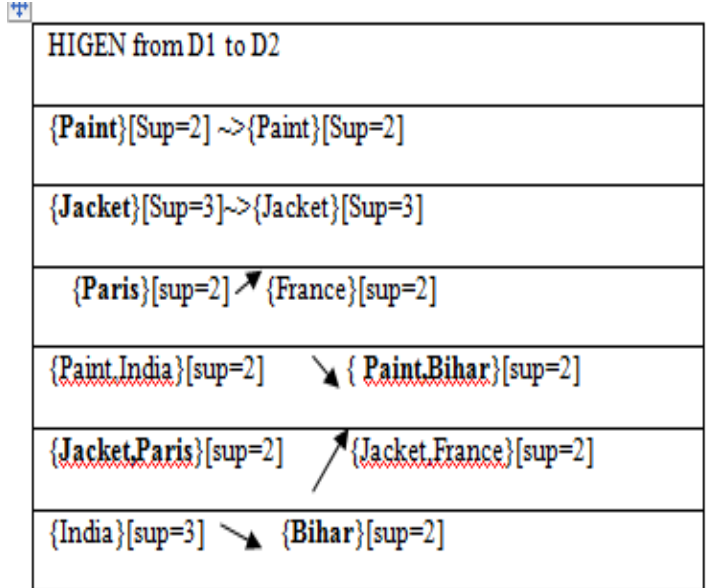

(b) Extracted HIGEN From D1 \& D2
(a)Generalized and not Generalized Itemsets mined min_sup=2

Extracted Patterns

\begin{tabular}{|c|c|c|}
\hline $\begin{array}{l}\text { Not Generalized } \\
\text { Itemset }\end{array}$ & Sup D1 & Sup D2 \\
\hline \{Bihar\} & 1(Inf.) & 2 \\
\hline \{Paris\} & 2 & 1 \\
\hline \{Paint\} & 2 & 2 \\
\hline \{Jacket\} & 3 & 3 \\
\hline \{Paint,Bihar\}\} & 1(Inf.) & 2 \\
\hline \{Iacket_Paris\} & 2 & 1 \\
\hline $\begin{array}{l}\text { Generalized } \\
\text { Itemset }\end{array}$ & Sup D1 & Sup D2 \\
\hline \{India\} & 2 & 3 \\
\hline \{France\} & 3 & 2 \\
\hline \{Paint.India $\}$ & 2 & 2 \\
\hline
\end{tabular}

We are using one dynamic pattern is History Generalized Pattern that is a higen represents the minimum sequence of generalizations required to keep information provided by a non generalized itemset which is frequent, with respect to the minimum support threshold, in each time duration. If an itemset is frequent in each time duration, the respected higen reports its support variations. Otherwise, when the itemset becomes infrequent at a specific point, the higen reports the minimum number of generalizations required to make its enclosed information frequent at a higher level of abstraction.frequent generalization at minimum abstraction level represents the information with minimal redundancy.

When an infrequent or not generalized itemset having multiple generalizations belonging to the same minimal aggregation level, number of higens associated with the same itemset are generated. Now focus the concentration of the analysts on the frequent generalizations of a uncommon itemset encolsing the same knowledge with a minimal amount of redundancy and, the reduced the number of the generated patterns. Type of higen algorithm is nonredundant higen, the frequent generalizations of the reference itemset of minimal generalization level distinguishd by minimal support.In Table 2 the set of HIGENs, which is mined from the given dataset by forcing an absolute minimum support threshold equal to 2 , is generated. The reference type of itemset is shown in table written in bold font. This arrow indicates means that the right side of itemset is a generalization of the left-side of itemset, \& involves a specializ changes occuers in the abstraction level. For, \{Jacket, Raxis $\quad$ \{Jacket, France $\}$ is a HIGEN stating that, from June (D1) to july (D2) 2013, the not generalized itemset Jacket,Paris \} is infrequent with respect to the minimum support threshold its upper level generalization as shown in the Fig 1 remains frequent. All the higens reported in Table 2 given to a different purpose not generalized reference itemset. The extraction process generate more higens each itemset, the generation of the nonredundant higens generates just the higens enclosing the minimal amount of redundancy, that is the ones including generalizations with minimal support. 


\section{RELATED WORK}

Literature survey of that concept is that in this topic used different data mining algorithm this algorithm having some limitations and some drawback.To overcome that drawback they have to used higen algorithm and non-redudandant higen algorithms are to be used.

1.Frequent itemset mining Algorithm:

The identification of sets of items, products, symptoms and characteristics, which occured together in the given database, is of the more fundamental tasks in Data Mining process. The aim of this algorithm is for searching frequent sets from the need to analyze also called as supermarket transaction data. We introduced mining association rules between sets of items in Large Databases the problem of mining association rules between sets of items in a large database of customer transactions. Each transaction consists of items purchased by a customer in a visit. finding those rules that have: The rules that we $\mathrm{f}$ have find item in the resultant and a union of any number of items in the antecedent. We solve this problem by decomposing it into two subproblems:

1. Finding all itemsets, called large itemsets, that are present in at least $s \%$ of transactions.

2. Generating from each large itemset, rules that use items from the large itemset.

steps: join and prune.

3. Info Miner Algorithm:

Effective mining algorithm, InfoMiner+, to simultaneously mine significant patterns and the associated subsequences. we introduced a new mining problem of partial periodic pattern with random replacement.[9]

\section{Existing Chatterbot system:}

Bot is an English simplification for the word robot, which is an agent which collaborates with a user or another program, simulating a human activity. The bots can be categorized in many categories, such as academics, searches, trades, etc; this deals with chatterbots or conversation robots.The goal of this type of bot is to answer and questions, in a particular way that people think they are talking to another person, instead of a computer program. The chatterbots have inside its knowledge base a set of simulated dialogs, to communicate with users in a natural language, and can be used as interfaces in a broad series of applications like electronic commerce, distance learning, among others. The chatterbots use Artificial Intelligence to simulate a dialog with a human being based in a "stimulus - response"routine: you make questions and he provides answers based on those questions. After a question is submitted in a natural language, the program queries a knowledge base and sends an answer trying to mimic the human behavior.

Prons

1. Temporal association rules are prone to become useless with the addition of new data to the database.

2. Reqiure less memory for storing dataset.

3. Minimum web data are required.

Cons

1. Privious chatter bot system is not updated to particular time being it only used exiting dataset

2. History oriented processing not available.

3. Processing and computation time are more.

4. Result never updated with time not upto use expectation.

\section{III. implementation details}

3.1 Design:

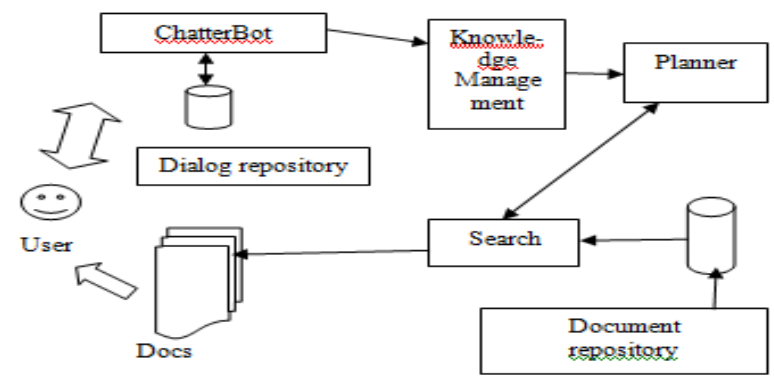

Fig III Chatter bot system

Objective of Project :

Find out temporal change pattern in mine by using real time dataset generation. Input and outcomes of project: We will use chat as web dataset then will train our system then will provide new set and detect change in it. All the work is done in Knowledge managament component Which is act as decision maker when we want 
to asked some question to that chat bot system it gives the answers but it discovered those are temporal change pattern or not then decision is made then and then it will provide the answers. We are developing New Chatterbot system which is better then privious chatter bot system this can be used any type of application and mining process is done in this system web dataset or live dataset is used as input. to this dynamic system with dynamic algotithm for example what's app,facebook chat is used as dataset. We are implementing the chatter bot system which previously implement but we are overcome the drawback of exiting system knowledge management is the main part in our system which is used to giving the answer to the pericular question and make the decision means mine that answer.

Block Diagram :

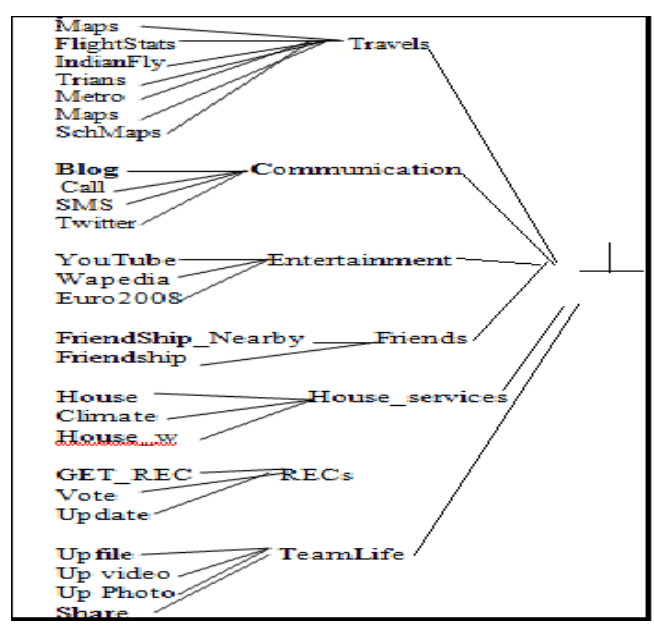

3.2 Algorithm :

Fig3.2 Generalization hierarchy over the Service attribute.

Algorithm 1. HIGEN MINER: HIstory GENeralized Pattern MINER-Input: Given ordered set of timestamped structured datasets $\mathrm{D}=\{\mathrm{D} 1 ; \mathrm{D} 2 ; \ldots ; \mathrm{Dn}\}$, minimum support threshold min_sup, taxonomy Output: set of HIGENs HG

$1: \mathrm{k} 1=1$ and and $\mathrm{HGN}=\varnothing ; / /$ Candidate length

2: $\mathrm{Ck}=$ set of distinct k-itemsets in $\mathrm{D}$ and repeat for all c $€ C k$ do scan Di and count the support sup(c,Di) Di $€$

$\mathrm{D}$ and end for $\mathrm{L}_{\mathrm{k}}^{\mathrm{i}}=\{$ itemsets $\mathrm{c} € \mathrm{Ckj} \sup (\mathrm{c}, \mathrm{Di})>=$ min_sup for some Di $€ \mathrm{D}\}$

3: HGN = update_HIGEN_set(Lik;HGN) 7:1 = $1 / /$ Candidate generalization level

$4: \mathrm{HGen}=\varnothing ; / /$ generalized itemset container and repeat for all c in Ck of level 1 do $10: \mathrm{D}^{\text {in }}{ }_{\mathrm{c}}=\{\mathrm{Di} € \mathrm{D}$ jsupðc;DiP < min_sup \} // datasets for which c is infrequent

5: if $\mathrm{D}^{\mathrm{in}} \neq \varnothing$; then $\operatorname{Hgen}(\mathrm{c})=$ set of new generalizations of itemset $\mathrm{c}$ of level $1+1$ then $\mathrm{Hgen}(\mathrm{c}$ )=taxonomy_evaluation $(0 ; c)$ for all Hgen $€ \operatorname{Hgen}(\mathrm{c})$ do Hgen.Desc $=\mathrm{c} / /$ Generalized itemset descendant and end for

6: HGen $=$ HGen U Hgen $(c)$ and end if , end for HGen $\neq \varnothing$; then for all Hgen $€$ HGen do scan Di and count the support of gen V Di $€ D_{\text {gen:Desc }}^{\text {inf }}$ for all Hgen $\mathrm{j} \sup \left(\right.$ Hgen,Di) $>=$ min_sup for some Di $€ D_{\text {gen:Desc }}^{\text {inf }}$ do HG1 = update_HIGEN_set(Hgen;HG1)

7: if $\sup \left(\right.$ Hgen,Di) $>=$ min_sup for all Di $€ \mathrm{D}_{\text {gen:Desc }}^{\text {inf }}$ then remove Hgen from HGen end if and end for and end for $\mathrm{Ck}=\mathrm{Ck} \mathrm{U}$ HGen and end if $1=1+1$ until HGen $=\varnothing ; \mathrm{k} 1=\mathrm{k} 1+1$ and $\mathrm{C}_{\mathrm{k}+1}=$ candidate_generation $\left(\mathrm{U}_{\mathrm{i}} \mathrm{C}_{\mathrm{k}}^{\mathrm{i}}\right)$ until $\mathrm{Ck}=\varnothing$; return $\mathrm{HG} 1[1]$

\section{ACKNOWLEDGMENT}

As wise person said about the nobility of the teaching profession, Catch a fish and you feed a man his dinner, but teach a man how to catch a fish, and you feed him for life. In this context, I would like to thank our helpful project guide Prof. Nawathe A.N. who had been an incessant source of inspiration and help. Not only did she inspire me to undertake this assignments, she also advise me throughout its course and help me during my times of trouble. I would like to thank H.O.D. of Department of Computer Engineering Prof. Paikrao R. L. for motivating me.Also, I would like to thank other member of Computer Engineering department who helped me to handle this assignment efficiently, and who were always ready togo out of their were to help us during our times of need.

\section{Conclusion}

This paper discovered the problem of change mining in the case of frequent itemsets. To represent the development of itemsets in different time periods without discarding relevant but rare knowledge due to 
minimum support threshold enforcement, it proposes to extract generalized itemsets characterized by minimal redundancy in this case one itemset becomes infrequent in a certain time duration. To this aim, two kinds of dynamic patterns, namely the Higens and the Nonredundant higens, have been introduced. [1]

\section{References}

[1] Discovering Temporal Change Patterns in the Presence of Taxonomies Luca Cagliero

[2] R. Agrawal, T. Imielinski, and A. Swami, "Mining Association Rules between Sets of Items in Large Databases," ACM SIGMOD Record, vol. 22, pp. 207-216, 1993.

[3] R. Agrawal and G. Psaila, “Active Data Mining,” Proc. First Int'l Conf. Knowledge Discovery and Data Mining, pp. 3-8, 1995.

[4] R. Agrawal and R. Srikant, "Mining Generalized Association Rules,” Proc. 21th Int'l Conf. Very Large Data Bases (VLDB '95),pp. 407-419, 1995.

[5] M.L. Antonie, O.R. Zaiane, and A. Coman, "Application of Data Mining Techniques for Medical Image Classification," Proc. Second Int'l Workshop Multimedia Data Mining (MDM/KDD '01), 2001.

[6] W.-H. Au and K.C.C. Chan, "Mining Changes in Association Rules: A Fuzzy Approach," Fuzzy Sets Systems, vol. 149, pp. 87104, Jan. 2005.

[7] E. Baralis, L. Cagliero, T. Cerquitelli, V. D’Elia, and P. Garza, “Support Driven Opportunistic Aggregation for Generalized Itemset Extraction," Proc. IEEE Fifth Int'1 Conf. Intelligent Systems (IS '10), 2010.

[8] S. Baron, M. Spiliopoulou, and O. Gnther, "Efficient Monitoring of Patterns in Data Mining Environments," Advances in Databases and Information Systems, L. Kalinichenko, R. Manthey, B. Thalheim,and U. Wloka, eds., vol. 2798, pp. 253-265, Springer, 2003.

[9] M. Bo" ttcher, D. Nauck, D. Ruta, and M. Spott, "Towards a Framework for Change Detection in Datasets," Research and Development in Intelligent Systems XXIII, M. Bramer, F. Coenen,and A. Tuson, eds., pp. 115-128, Springer, 2007.

[10] D.W.-L. Cheung, J. Han, V. Ng, and C.Y. Wong, "Maintenance of Discovered Association Rules in Large Databases: An Incremental Updating Technique,” Proc. 12th Int'l Conf. Data Eng. (ICDE '96),pp. 106-114, 1996.

[11] C. Clifton, R. Cooley, and J. Rennie, "Topcat: Data Mining for Topic Identification in a Text Corpus," Proc. Third European Conf. Principles and Practice of Knowledge Discovery in Databases,2002.

[12] G. Dong, J. Han, J.M.W. Lam, J. Pei, K. Wang, and W. Zou, "Mining Constrained Gradients in Large Databases," IEEE Trans.Knowledge and Data Eng., vol. 16, no. 8, pp. 922-938, Aug. 2004.

[13] G. Dong and J. Li, "Mining Border Descriptions of Emerging Patterns from Dataset Pairs," Knowledge and Information Systems,vol. 8, pp. 178-202, Aug. 2005.

[14] B. Liu, W. Hsu, and Y. Ma, "Discovering the set of Fundamental Rule Changes," Proc. Seventh ACM SIGKDD Int'l Conf. Knowledge Discovery and Data Mining, pp. 335-340, 2001. 